\section{AUS ERSTER HAND}

\section{Chancen für eine höhere Rohstoffausbeute im Bergbau}

Die Europäische Union muss nach eigenen Angaben jährlich ca. 200 Mio. t Mineralien importieren, obwohl in stillgelegten Bergwerken oder kleineren Lagerstätten in einer Tiefe von 500 bis $1000 \mathrm{~m}$ noch Bodenschätze im Wert von etwa $100 \mathrm{Mrd}$. € liegen sollen. Aber der Abbau mit herkömmlicher Technik ist offensichtlich nicht lohnenswert. Bekanntlich gibt es im Erzgebirge auch immer wieder Aktivitäten, seltene Erden zu fördern. Die Ressourcen liegen oft auch in gefluteten Altbergwerken oder in seichten Gewässern. Deshalb wird aktuell von der EU das Forschungsprojekt Vamos (Viable Alternative Mine Operating System) mit einem Umfang von 12,6 Mio. $€$ und einer Laufzeit bis Mitte 2018 gefördert. Hauptgegenstand ist ein ferngesteuerter Spezialbagger, der Rohstoffe unter Wasser finden und abbauen soll. Der Prototyp soll bereits fertig gestellt sein für die vorgesehene Erprobung an 4 geeigneten Standorten in Europa.

Der nächste Entwicklungssprung im Bergbau wird wohl ohnehin von der „Robolution“ geprägt sein. Das heißt, Roboter sollen an gefährlichen, lauten und schmutzigen Orten effizienter arbeiten als die Bergleute. Menschen sollen hingegen Umweltbedingungen überwachen und bisher unerreichte Rohstoffe gewinnen. Roboter werden bereits heute in modernen und gut ausgebauten Bergwerken, wie z.B. im Salzabbau, eingesetzt. Für engere Erzgruben bestanden bisher keine brauchbaren Lösungen. An der TU Bergakademie Freiberg wird am Institut für Informatik an der Zukunft des Bergbaus gearbeitet. Dazu werden Spezialroboter verschiedener Größen bis $140 \mathrm{~kg}$ einer kanadischen Firma unter Tage erprobt. Als Versuchsfeld dienen die engen Gänge der nahe gelegenen "Reichen Zeche", ein über mehrere Jahrhunderte betriebenes Bergwerk, welches heute als Lehr-Forschungsbergwerk $147 \mathrm{~m}$ unter der Erde dient. Die Roboter bewegen sich selbständig auf Rädern mit Hilfe von Laserscannern durch die Gänge. Es können u.a. 3D-Bilder aufgezeichnet werden. Ein Roboter verfügt auch über einen Greifarm, der einen Scanner halten kann, um per Röntgenfluoreszenzanalyse Gesteine zu bestimmen. Der Greifarm soll außerdem WLAN-Router zur Orientierung platzieren. Vorgesehen ist auch die Aufnahme wichtiger Daten, wie Strahlung, Temperatur, Luftdruck und Gaskonzentration.

Der Blick in die Zukunft des Bergbaus könnte die komplette Steuerung aus der Ferne sein. Neben der Ersparnis menschlicher Arbeitskräfte, würde die Effizienz der Förderung erhöht werden. Im Tagebau ist das bekanntlich einfacher. Dort können autonome Fahrzeuge auch GPS nutzen und die Automatisierung ist heute bereits weiter gediehen. Im Untertagebau hingegen sollen z.Zt. die technischen Kapazitäten durchschnittlich nur zu $25 \%$ ausgelastet sein. Hinzu kämen Ersparnisse bei der Bewitterung der Gruben. Vom Institut für Bergbau an der TU Clausthal wird hinsichtlich einer möglichen Umsatzsteigerung durch Robotertechnik im Untertagebau als „eine Frage des Preises“ bezeichnet. Wo Lohn und Rohstoffpreise hoch seien, könnten sich die Investitionen auszahlen. Die Forscher an der Bergakademie Freiberg verfolgen allerdings noch ein weiteres Ziel, nämlich historische Schächte. Bevor diese wegen Einsturzgefahr geschlossen werden müssen, können sie mit Roboter und Kamera für die Nachwelt virtuell zugänglich bleiben.

M. Röhrs

\section{Additive Fertigung: Forschungs- felder und Ausbildung im Fokus des DVS}

Der Fachausschuss "Generative Fertigungsverfahren - Rapidtechnologien“ innerhalb des DVS - Deutscher Verband für Schweißen und verwandte Verfahren e. V. in Düsseldorf befasst sich intensiv mit den Forschungsfeldern, die die gesamte Prozesskette der additiven Fertigung betreffen. Dabei stehen die Technologieentwicklung, die Steigerung der Akzeptanz zur Nutzung dieser Technologie bei KMU (kleine und mittlere Unternehmen) und das Schaffen neuer Anwendungsbereiche im Vordergrund. Neue Mitglieder sind immer herzlich willkommen. Zurzeit setzt sich das Gremium aus über 50 führenden Experten aus Forschung und Industrie zusammen.

\section{Bildung und Zertifizierung}

Die Fachgruppe „Ausbildung in der additiven Fertigung" des Ausschusses für Bildung im DVS entwickelt derzeit Ausbildungskonzepte in den Fachrichtungen „Kunststoff und Metall".

Eine „Fachkraft für additive Fertigungsverfahren" soll ein systematisches Überblickwissen besitzen sowie die Fachbegriffe, die Prozesskette, die Möglichkeiten und Grenzen der Verfahren in Bezug auf Qualität, Kosten und Anwendungsgebiete kennen. Sie bedient die zur Verfügung stehende Anlagentechnik und kann beispielhaft ein Bauteil herstellen. Unternehmen, die über entsprechende Fachkräfte verfügen, profitieren von unerlässlichen Kenntnissen für eine moderne Fertigung.

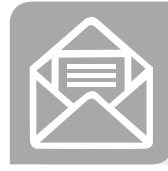

Nähere Informationen zu Forschung \& Technik: www.dvs-forschung.de/FA13

Informationen zu Bildungseinrichtungen:

www.dvs-bildungseinrichtungen.de

\section{Weitere Förderung für TUHH Sonderforschungsbereich}

Der Sonderforschungsbereich SFB 986 „Maßgeschneiderte Multiskalige Materialsysteme - M3" wird für weitere vier Jahre von der Deutschen Forschungsgemeinschaft (DFG) gefördert. Der SFB 986 ist eine Kooperation der Technischen Universität Hamburg (TUHH), des Helmholtz-Zentrum Geesthacht (HZG), der Universität Hamburg (UHH) und des Deutschen Elektronen-Synchrotrons (DESY). Das Gesamtvolumen der Förderung beträgt 13 Millionen Euro. Start der zweiten Förderphase ist der 1. Juli 2016. „Der Fortbestand des SFB zeigt, dass wir materialwissenschaftliche Spitzenforschung auf internationalem Niveau betreiben", so Professor Dr. Gerold Schneider, Sprecher des SFB 986 und Leiter des TUHH-Instituts für Keramische Hochleistungswerkstoffe. 